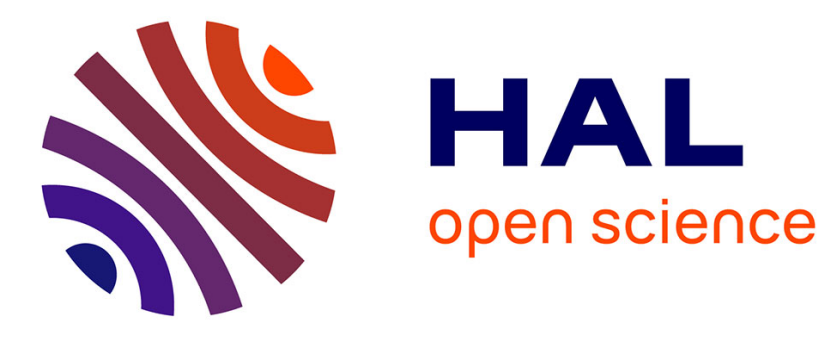

\title{
Penetratin translocation mechanism through asymmetric droplet interface bilayers
}

\author{
P. Gehan, Simon Kulifaj, P. Soule, J.B. Bodin, Mehdi Amoura, Astrid \\ Walrant, Sandrine Sagan, Abdou Rachid Thiam, Kieu Ngo, Vincent Vivier, et \\ al.
}

\section{To cite this version:}

P. Gehan, Simon Kulifaj, P. Soule, J.B. Bodin, Mehdi Amoura, et al.. Penetratin translocation mechanism through asymmetric droplet interface bilayers. BBA-Biomembranes, 2020, 1862, pp.183415. 10.1016/j.bbamem.2020.183415 . hal-02910851

\section{HAL Id: hal-02910851 https: / hal.sorbonne-universite.fr/hal-02910851}

Submitted on 3 Aug 2020

HAL is a multi-disciplinary open access archive for the deposit and dissemination of scientific research documents, whether they are published or not. The documents may come from teaching and research institutions in France or abroad, or from public or private research centers.
L'archive ouverte pluridisciplinaire HAL, est destinée au dépôt et à la diffusion de documents scientifiques de niveau recherche, publiés ou non, émanant des établissements d'enseignement et de recherche français ou étrangers, des laboratoires publics ou privés. 
Penetratin translocation mechanism through asymmetric droplet interface bilayers

Gehan* P, Kulifaj* S, Soule P, Bodin JB, Amoura M, Walrant A, Sagan S, Thiam AR, Ngo K, Vivier V, Cribier S, Rodriguez N

*: these two authors contributed equally 


\section{ABSTRACT}

Penetratin is a cell penetrating peptide (CPP) that can enter cells by direct translocation through the plasma membrane. The molecular mechanism of this translocation still remains poorly understood. Here we provide insights on this mechanism by studying the direct translocation of the peptide across model membranes based on Droplet Interface Bilayers (DIBs), which are bilayers at the interface between two adhering aqueous-in-oil droplets. We first showed with symmetric bilayers made of a mix of 1-palmitoyl-2-oleoyl-sn-glycero-3-phospho-(1'-racglycerol) (POPG) and 1,2-dioleoyl-sn-glycero-3-phosphocholine (POPC) that the translocation of penetratin required the presence of at least $40 \%$ of POPG on both leaflets. Interestingly when replacing POPG with another anionic lipid 1-palmitoyl-2-oleoyl-sn-glycero-3-phospho-Lserine (POPS), translocation was inefficient. To elucidate the lipid partners required at each step of the CPP translocation process, we then investigated the crossing of asymmetric bilayers. We found that POPG on the proximal leaflet and POPS on the distal leaflet allowed penetratin translocation. Translocation was not observed when POPS was on the proximal leaflet and POPG on the distal leaflet or if POPS on the distal leaflet was replaced with POPC. These observations led us to propose a three-step translocation mechanism: (i) peptide recruitment by anionic lipids, (ii) formation of a transient peptide-lipid structure leading to the initiation of translocation which required specifically POPG on the proximal leaflet, (iii) termination of the translocation process favored by a driving force provided by anionic lipids in the distal leaflet.

\section{INTRODUCTION}

\section{CPP translocation through bilayers}

Cell penetrating peptides (CPPs) are cationic peptides that possess the ability to enter into cells. They can be used to drag exogenous molecules such as proteins, nucleotides or drugs and are thus very promising for the field of vectorization (1-4). In the literature, CPPs are demonstrated to enter cells by two different general mechanisms: endocytosis and direct translocation through the plasma membrane. The relative importance of these two pathways depends, among other factors, on the amino acid content of the CPP (5). Even when direct translocation is not the major path of entry, its importance for vectorization is crucial because it allows the CPP and its cargos to end up directly into the cytoplasm. In this case, there is no need for the cargo to escape endosomes to reach its targets in the cytoplasm. 
The molecular mechanism of the direct translocation of CPPs is currently little understood. Intermediate peptide-lipid structures have been hypothesized as inverted micelles, pores or lipid-peptide complexes (6). All these models propose an explanation for the unexpected ability of a rather hydrophilic CPP, soluble in aqueous media, to cross the hydrophobic core of the lipid bilayer. The present study tries to answer two classes of questions related to the comprehensive analysis of the direct translocation mechanism in model membranes as a prerequisite step to the study of cell membrane translocation: (i) Which are the lipid partners favoring direct translocation and do negatively charged lipids play a particular role in this mechanism due to favorable interactions with positively charged CPPs? (ii) What are the driving forces that lead the CPPs to adhere, locally destabilize and then cross the lipid bilayer? Can the negatively charged lipids be involved in this driving force and does their localization on one or the other leaflet of the bilayer play a role in these driving forces?

\section{Model membranes to study translocation}

Different model membranes composed exclusively of lipids have been used to study the passive translocation mechanism since it is very likely to involve the crossing through the lipid bilayer of the plasma membrane.

Vesicles of various compositions have been used to determine the lipid partners that favor direct translocation (7-15). Previously, Walrant et al. used an original mass spectrometry based protocol to quantify the internalization of CPPs inside liposomes and showed the importance of the negatively charged lipids to activate translocation. In addition Swiecicki et al. designed a protocol based on the quenching of fluorescently labeled CPPs outside of vesicles to determine the fraction of CPP able to translocate into vesicles. Using this method, they showed the importance of the negatively charged phosphatidylglycerol (PG) lipid to favor the translocation. Finally, Herce et al. have used planar suspended bilayers to study the translocation of CPPs (16). In this latter work, the crossing of the CPP could not be detected but its impact on the membrane (formation of pores) was evidenced.

Droplet interface bilayer (DIB) is also used as a model membrane (17). To form a DIB, two aqueous droplets in oil covered by a lipid monolayer are brought into contact and the interface of these two droplets constitutes a bilayer. Two main techniques exist to bring the lipids at the oil-water interface. With the "lipid in" technique (17), lipids are introduced in the form of vesicles in the aqueous phase that will collapse at the oil-water interface and bring the lipids. With the "lipid out" technique (18), lipids are introduced in the oil phase, their mixing being possibly favored by adding to the oil a good lipid solvent such as chloroform. Several methods 
are then available to form the individual droplets and bring two of them into contact to get a DIB at the interface: gentle mixing, use of a capillary micropipette, microfluidic devices, etc. Depending on various parameters such as lipid and solvent natures, lipid good solvent and oil ratio, lipid concentration, lipid in or lipid out method, evaporation conditions or droplet sizes the DIB stability can range from seconds to hours. This DIB model membrane can be used to study the transport of molecules through bilayers (19-21). This model system has been used by Li et al. to evidence that the crossing of a bilayer by the CPP Pep1 is driven by the presence of negatively charged PG lipids on the distal leaflet (the leaflet which is not initially in contact with Pep1 as opposed to the proximal leaflet) (21). Although negatively charged lipids are minor components of the outer leaflet of cell plasma membrane (22), it is currently assumed that negative charges (as found in the extracellular matrix) at the cell surface are crucial to attract/concentrate cationic peptides in the close vicinity of the lipid bilayer (6). Thus, the negatively charged lipids we used herein also mimic the negatively charge environment found at the cell surface.

\section{The case of the CPP penetratin}

Penetratin peptide is among the first discovered CPPs (23). Its sequence is included in the sequence of the larger Antennapedia homeoprotein. Penetratin is a cationic peptide but with an amphipathic nature due to the presence of two hydrophobic tryptophans. In contact with a membrane it can adopt an alpha-helical or beta-strand secondary structure (24-26).

Penetratin is known to enter partly by direct translocation, for example into Chinese Hamster Ovary $(\mathrm{CHO})$ cells (5). After incubation of cells for $1 \mathrm{~h}$ with $5 \mu \mathrm{M}$ penetratin, Jiao et al. estimated that $30 \%$ of the penetratin entered by direct translocation, whereas the remaining $70 \%$ used endocytosis pathways. It is thus interesting to focus on this peptide to determine the membrane lipid components and their localization on both leaflets that are crucial for translocation. Here, we have used DIB to investigate the membrane compositions favoring the translocation of penetratin. For this purpose, penetratin was labeled with a fluorophore cargo molecule, which allowed detection and tracking of the peptide. 


\section{MATERIALS AND METHODS}

\section{Chemicals}

Magnesium chloride $\left(\mathrm{MgCl}_{2}\right)$ and squalene were purchased from Sigma Aldrich. PBS was obtained from Merck. Chloroform was obtained from Carlo Erba. All chemicals were used as received without further purification.

\section{Lipids}

All the lipids, dissolved in chloroform to obtain a concentration of $25 \mathrm{mg} / \mathrm{ml}$, were purchased from Avanti Polar Lipids: 1-palmitoyl-2-oleoyl-sn-glycero-3-phospho-(1'-rac-glycerol) (POPG), 1-palmitoyl-2-oleoyl-sn-glycero-3-phosphocholine (POPC), 1-palmitoyl-2-oleoyl-snglycero-3-phospho-L-serine (POPS), 1,2-dioleoyl-sn-glycero-3-phosphocholine (DOPC), 1,2dioleoyl-sn-glycero-3-phospho-L-serine (DOPS).

\section{Peptides and fluorophores}

Two peptides have been used in this study: penetratin (RQIKIWFQNRRMKWKK) and RL9 (RRLLRRLRR) taken as a negative control of translocation (27). Both were chemically labeled with a fluorophore on their N-terminal part. A green fluorophore has been used: Alexa488 maleimide (Life technologies) introduced on the side chain of an additional Cysteine on the Nterminus.

Peptides were synthesized in our laboratory by solid-phase synthesis using the Boc (penetratin) or Fmoc (RL9) strategy. They were purified by RP-HPLC and their structure further checked by mass spectrometry.

FluoProbe FP488 was purchased from Interchim.

\section{DIB formation}

DIBs were formed at the interface between two aqueous droplets in oil. Two methods were used in this study in order to form DIB:

(i) Peptide translocation assay

Two populations of aqueous droplets in oil were mixed. Aqueous phases were prepared in PBS containing $10 \mathrm{mM} \mathrm{MgCl}_{2}$ to favor DIB stability. One of the populations additionally contained $5 \mu \mathrm{M}$ of peptide in its aqueous phase. The oil phase was prepared with $15 \%(\mathrm{v} / \mathrm{v})$ of chloroform and $8 \% 000(\mathrm{w} / \mathrm{w})$ of lipids in squalene. Each population of droplets was prepared with $2 \mu \mathrm{L}$ of 
aqueous phase mixed with $20 \mu \mathrm{L}$ of the oil phase. The sample was briefly shaken to produce the invert emulsion. Two populations of droplets were mixed on a glass slide covered by a thin layer of PDMS (used to avoid spreading of the droplets on the glass slide), thus leading to randomly formed pairs. An at most very low presence of oil in the DIB has been suggested by electrical measurements of Gross et al. (28). This is different from what is obtained with giant vesicles formed by the water in oil emulsion method (29). The fluorescence signal was recorded over 30min with a Princeton Instrument Micromax camera and an IX71 Olympus inverted microscope equipped with 10x or 20x Olympus objectives. The fluorescent observations were made using an HBO lamp and a fluorescence cube with an excitation filter Omega XF18-2 $(457-487 \mathrm{~nm})$.

(ii) Asymmetric bilayer stability assay

The DIBs were formed and observed similarly as in the peptide translocation assay, but in the absence of peptide in the aqueous phases of the two droplet populations. In that case, the aqueous phase of one population contained the fluorescently labeled (with fluoprobe Alexa488) annexin $\mathrm{V}$ (from Life Technologies, reference A13201 diluted 10 times) and $2.5 \mathrm{mM} \mathrm{CaCl}_{2}$.

\section{Peptide detection}

The epifluorescence images of a pair of adhering droplets could be used to detect the fluorescently labeled peptides in both droplets.

The quantification of fluorescence is made by averaging the fluorescence on a disk centered on the droplet (the diameter of the disk is roughly half the diameter of the droplet). This disk does not include the edges which are brighter in certain conditions. However, because our technique is epifluorescence microscopy, the fluorescence we measure in this disk integrates a contribution from peptides bound at the top and bottom edges of the droplet.

\section{RESULTS}

\section{Symmetric bilayers bearing enough POPG allow penetratin translocation}

The ability of the fluorescently labeled penetratin (fluo-penetratin) to translocate through a strongly negatively charged bilayer made of 100\% POPG lipids was first assessed. Fluopenetratin at $5 \mu \mathrm{M}$ was initially present in one of the two droplets forming a pair. The increase of fluorescence in the initially peptide-free droplet was followed for 30 minutes and was 
considered as the signature for the translocation of fluo-penetratin through the POPG DIB. Epifluorescence and bright field images of the droplets are shown in Figure 1A. The initially dark droplet (initially containing no fluo-penetratin) became significantly fluorescent after a few minutes. The stronger fluorescence on the monolayer and DIB of the droplets was likely due to the favorable electrostatic interaction between fluo-penetratin and POPG favoring the adhesion of peptides on lipids. The dye of fluo-penetratin adhering on the monolayer or the DIB may also become brighter due to a different surrounding, which may also explain partly this brightness of the rim. It is noteworthy that the equality of fluorescence intensities between the two droplets was not reached after 30 minutes. For longer experiments this equilibrium between the two droplets was never reached and a fluorescence plateau was often observed. Figure 1B shows the kinetics of crossing measured in the experiments of figure 1A. The time for crossing is of the order of a few minutes. Sometimes we observed that the crossing started after a few minutes delay. A systematic analysis of the crossing kinetics of fluo-penetratin is presented in Supplementary Material, Figure S1. In order to prove that the crossing of penetratin through the DIB that we monitored was related to the cell-penetration capacity of fluopenetratin, the membrane-binding but non cell-penetrating RL9 peptide was tested as a negative control. The fluo-RL9 peptide is positively charged and rich in arginine residues as the fluopenetratin, and has been shown to be a poor CPP which adheres to membranes but does hardly cross cell or vesicle membranes (11). A typical experiment with $5 \mu \mathrm{M}$ fluo-RL9 is shown in Figure 1A. The adhesion of fluo-RL9 on the monolayer and DIB was also detected. After 30 minutes however no significant fluorescence appeared in the initially fluo-RL9 free droplet showing that fluo-RL9 did not translocate during this experiment.

These fluo-penetratin and fluo-RL9 translocation trials through 100\% POPG symmetric DIBs were reproduced several times. Interestingly the final fluo-penetratin fluorescence intensities in the initially peptide free droplet after 30 minutes varied from one trial to the other and for 2 trials out of 12 no translocation at all was detected (figure 1C). Two populations of experiments are thus observed. These results showed that translocation is a stochastic phenomenon (which can occur or not), certainly dependent of the formation of one or several transient peptide-lipid permeation structures whose formation probability and lifetime seems to be stochastic. The implications of this stochasticity on the translocation mechanism are further discussed in the discussion section. This stochasticity leads us to describe these translocation experiments in probabilistic terms in order to try to characterize the probability of occurrence of the transient permeation structure. We thus set the criterion that translocation indeed occurred if we could detect a significant fluorescence of the droplet initially free of peptide within 30 minutes. This 
criterion can be expressed in terms of fluorescence: we assumed that a significant translocation happened if the initially dark droplet had reached at least $10 \%$ of the fluorescence of the other droplet after 30 minutes. For all the following translocation experiments this criterion was used to determine the translocation probability namely the proportion of experiments for which a significant translocation was detected.

The negatively charged POPG seemed to play an important role in the translocation. In order to further address this role, different POPG:POPC ratios were used in the preparation of symmetric DIBs. We measured then the translocation probability of fluo-pentratin with various proportions of POPG in the POPG:POPC DIB as shown on Figure 2. For a proportion of POPG in POPC equal to or above $40 \%$ of the total amount of lipids, a translocation probability above $75 \%$ was detected for fluo-penetratin. It was significantly different from the low translocation probability of RL9, found to be below $20 \%$, thus showing that the observed crossing must be ascribed to the cell-penetrating ability of the fluo-penetratin. We thus concluded that a threshold level of $40 \%$ negatively charged POPG lipids in the DIB with which fluo-penetratin can interact, is necessary for the peptide to translocate through symmetric bilayers.

To determine whether this observed translocation of fluo-penetratin could be ascribed to certain characteristics of our model membrane, for example the occurrence of transient instabilities, we have also performed translocation tests of soluble fluorescent probe (FP488). These tests have been performed for different membrane compositions and the results are presented on figure 3. For a proportion of POPG in the POPG:POPC bilayer above $40 \%$, translocation of FP488 was never observed. However, some permeation of FP488 through DIB containing enough POPC were detected. It is nevertheless important to notice that these instabilities of our model membranes occurred only for these lipid compositions for which no translocation of penetratin was detected and could thus not explain the membrane crossing behavior of penetratin through the DIBs. The systematic use of the RL9 negative control is another guarantee that the observed fluo-penetratin tranlocation events for certain lipid compositions are indeed due to penetratin cell penetrating ability and not due to transient bilayer instability.

\section{Role of the fluo-penetratin concentration}

We then checked for a possible effect of the fluo-penetratin concentration on the probability of the translocation process. For this purpose, experiments with $100 \%$ POPG DIBs and $2 \mu \mathrm{M}$ fluo-penetratin in the droplet initially containing the peptides were designed. Results are shown on Figure 4. The probability of occurrence of translocation of fluo-penetratin is strongly 
reduced at $2 \mu \mathrm{M}$ as compared to the probability at $5 \mu \mathrm{M}$. This result is consistent with a concentration-dependent step during the translocation process of fluo-penetratin. This step is likely to imply several fluo-penetratins destabilizing collectively the bilayer. Indeed, if fluopenetratin peptides would cross the DIB without interacting at any stage, this mechanism would lead to a translocated peptide amount at low concentration with a similar kinetics as at high concentration and a translocated amount proportional to the concentration. This is not what we have observed since in our conditions fluo-penetratin at low concentration does not translocate less but does not translocate at all (not significantly different from the negative control fluoRL9).

Charge is not enough: the negatively charged POPS lipid does not enable penetratin translocation through symmetric POPG bilayer

Strong electrostatic interactions between POPG and penetratin may drive its adhesion and then translocation across the bilayer. Assuming that the interaction is purely electrostatic, it should be independent of the chemical nature of the lipid head group bearing the negative charge. To test this hypothesis, POPG was substituted with another anionic lipid: POPS. We first observed that the adhesion of the droplets was less favorable because the two droplets forming a pair did not flatten within tens of minutes to reach hemispherical shapes as was previously observed with POPG. This dependence of the shape of a pair of adhering droplets upon the nature of the lipids has already been described by Ben M'barek et al (30). The flattening of the two droplets is favored when the adhesion energy of two lipids monolayer in oil (with tails facing the oil) is higher and impaired when the water-oil interface covered by a monolayer of lipid has a higher surface tension (20). These two parameters are likely to be different for POPG and POPS lipids and certainly explain the difference of shape of the POPG and POPS droplet pairs.

Regarding the translocation, we tested the fluo-penetratin translocation on five DIBs and only one experiment out of these five lead to the observation of a detectable translocation of fluopenetratin through the $100 \%$ POPS bilayer (Figure 5B): this proportion was different from the translocation probability through a 100\% POPG bilayer and not significantly different from the translocation probability of fluo-RL9 through a 100\% POPS bilayer. This absence of translocation cannot be ascribed to a lack of adhesion of the fluo-penetratin on the DIB as evidenced by the picture where the fluorescence of the DIB and monolayer of the droplet initially containing fluo-penetratin is clearly visible (Figure 5A). It thus seemed that charge is not enough and that the nature of the interactions between the peptide and lipid, beside 
electrostatics, plays a role in favoring its translocation. It is also noticeable that these interactions take place in the presence of divalent magnesium cations $(5 \mathrm{mM})$. The physicochemical properties of POPG and POPS are modified in the presence of magnesium $(31,32)$ in a way that may impact their interactions with fluo-penetratin.

Characterization of the asymmetric DIBs: asymmetry can be maintained for $\mathbf{4 5}$ minutes The biological bilayers are asymmetric with different lipid compositions in their two leaflets (33). To go further to better mimic biological bilayers and decompose the peptide translocation mechanism in steps corresponding to its interaction with each lipid leaflet of the bilayers we conducted translocation experiments on asymmetric DIBs. Asymmetric DIB have already been obtained by another method (34). In our study, asymmetric DIBs could be obtained by mixing two populations of droplets with different lipid compositions. When two droplets from different populations adhered, the DIB was asymmetric with each leaflet having the lipid composition of its population. We had first to check whether this asymmetry is maintained with time since we cannot discard the possibility of events such as exchanges between the lipids in the droplets and the mixed lipids in the surrounding oil phase or flip-flop of the lipids in the DIB. To test these hypotheses, we investigated the possible appearance of DOPS lipids on a droplet surrounded by DOPC and adhering to a droplet surrounded by DOPS. We used fluorescently labeled annexin $\mathrm{V}$ (fluo-annexin $\mathrm{V}$ ) in the presence of $2.5 \mathrm{mM} \mathrm{CaCl}_{2}$ since annexin $\mathrm{V}$ is known to bind specifically to PS phospholipids. In this condition, the monolayer of the initially DOPC covered droplet can become fluorescent if enough DOPS access to the monolayer of this droplet (which means that asymmetry is partially lost). In a preliminary set of experiments, the sensitivity of this detection was investigated and it was shown that about $10 \%$ of DOPS was required to have a detectable fluorescent monolayer (see supplementary Figure 2). We tracked four DOPCDOPS pairs containing fluo-annexin $\mathrm{V}$ in the DOPC droplet for which no fluorescent monolayers could be observed over a 45 min time duration (Figure 6). These experiments indicated that the asymmetry of the bilayer was stable for at least $45 \mathrm{~min}$ in our model system for a DOPC/DOPS asymmetric bilayer. We hypothesized that this was also true for POPC or POPS/POPG bilayers, which was confirmed by the observation of the adhesion or not of the peptide on the monolayers as explained below. 
Fluo-penetratin does not cross proximal:distal POPG:POPC or POPC:POPG asymmetric bilayers

The possible translocation of fluo-penetratin though asymmetric bilayers formed by POPG in one leaflet and POPC in the other was tested with peptides initially either in the POPG or POPC side (the initial side of the peptide is named proximal, the other side distal). Typical experiments are shown in Figure 7 and the results are summarized in Table 1. It is noticeable that when fluopenetratin was present in the POPC droplet, the monolayer of the proximal droplet was not fluorescent, which confirmed that negatively charged lipids favor the recruitment of the peptides at the membrane. It is also noticeable that this absence of fluorescence was maintained over time when the POPC droplet adhered to a POPG droplet which is consistent with an absence of appearance of POPG on the monolayer of the POPC droplet: this further evidenced the conservation of the lipid asymmetry between leaflets in the bilayer in our model. Regarding the fluo-penetratin translocation, in both conditions (fluo-penetratin in the POPC or in the POPG droplet) no translocation of fluo-penetratin was observed. This result demonstrated that POPG on the proximal or distal leaflet was not enough for fluo-penetratin translocation.

Fluo-penetratin crossed proximal:distal POPG:POPS but not POPS:POPG asymmetric bilayers

The absence of translocation of fluo-penetratin through asymmetric proximal:distal POPG:POPC DIBs whereas the peptide crossed symmetric POPG DIBs may be ascribed to the absence of an anionic lipid on the distal leaflet to drive the peptide translocation towards the distal leaflet and then the free droplet. We showed indeed that fluo-penetratin translocated through proximal:distal POPG:POPS bilayers (Figure 8) confirming that negatively charged lipids on the distal leaflet such as POPG (in the symmetric bilayer experiments) or POPS can drive the completion of the translocation process. On the other hand, fluo-penetratin did not translocate through proximal:distal POPS:POPG asymmetric DIBs. This result showed the necessity of the specific presence of POPG on the proximal leaflet to drive the fluo-penetratin translocation: for this step of the translocation, which follows adhesion, charge is not enough and the specific chemical nature of POPG is required. 


\section{DISCUSSION}

\section{The stochasticity of the translocation}

We observed that for a given condition (lipid composition) we could observe different behaviors of fluo-penetratin as far as translocation is concerned: significant translocation (obtained when the initially dark droplet reaches at least 10\% of the fluorescence signal of the fluo-peptide containing droplet after 30 minutes) could be observed or not. This stochastic nature of the translocation phenomenon is not what we would have expected from peptides individually interacting and crossing the membrane. Indeed it was expected that the behavior of the numerous peptides involved would have been averaged to a reproducible overall translocation rate. But we did not observe this average behavior and instead of determining a permeability coefficient for fluo-penetratin, we have searched to quantify a probability of translocation. We interpret this stochasticity of translocation as a dependence of this phenomenon on a specific event that can occur or not and trigger the translocation of some peptides. This event corresponds to the formation of a transient peptide-lipid structure that favors translocation. Different peptide-lipid structures have been proposed to explain the translocation of penetratin such as inverted micelles or pores $(6,13,35,36)$. The formation of this structure which lead to translocation is further suggested by the observed concentration dependence of the translocation process which is consistent with several peptides destabilizing the bilayer to enable the translocation.

\section{A three step translocation mechanism for penetratin}

Altogether, these results showed that in these experiments: (i) POPG or POPS on the proximal leaflet leads to an accumulation of fluo-penetratin on the bilayers as assessed by the observed fluorescence of the bilayer in the presence of these lipids (ii) negatively charged POPG lipids were always necessary on the proximal leaflet of the bilayer to favor fluo-penetratin translocation (iii) POPG or POPS on the distal leaflet could drive fluo-penetratin translocation. These conclusions lead us to propose a three-step mechanism for cationic peptide translocation. A first step consists in the accumulation of peptides on the membrane driven by recruitment of peptides by anionic lipids. A second step is the insertion/destabilization of the membrane that is initiated by the interaction of the fluo-penetratin with the lipids of the proximal leaflet. This step leads to the formation of a transient peptide-lipid structure that will enable the translocation. For this second step specific lipids are necessary as evidenced by the necessity of POPG as compared with POPS on the proximal leaflet. The third step is the completion of 
the crossing which needs a driving force that can be provided by the presence of anionic POPG or POPS on the distal leaflet.

\section{Different roles for POPS and POPG in the penetratin translocation process}

We observed that for both the symmetric and asymmetric DIB POPG could not simply be replaced by POPS and that the latter was less favorable to fluo-penetratin translocation. It thus emphasized that charge is not enough. Additional differences may come from: (i) Peptide-lipid interactions are not simply related to the number of charges and the chemical nature of the chemical groups bearing these charges may matter by impacting the electrostatic interactions or even through more complicated kind of interactions (steric for example) (ii) the transient peptide-lipid structures formed to start the translocation process depend from properties of the lipid different from its charge, for example its shape, that may favor or impair the formation of such structures.

\section{CONCLUSION}

With the DIB model that gives us access to asymmetric bilayers, we have evidenced that negatively charged lipids are not equivalent. Indeed, POPG lipids were necessary in the proximal leaflet of the bilayer to favor the translocation of penetratin and penetratin and lipids likely form transient peptide-lipid structures that enable this translocation. Anionic lipids in the proximal leaflet are likely to favor the accumulation of peptides, while anionic lipids in the distal leaflet appear to provide a driving force for the crossing of penetratin. Since biological cell membranes of mammals do not contain PG lipid but many other negatively charged lipids, further investigation is required to identify those recruited by penetratin to translocate.

\section{AUTHOR CONTRIBUTIONS}

SK, PG, PS, JBB, MA and AW performed the experiments. SK, PG, PS, JBB analyzed the data. SS, ART, KN, VV, SC and NR designed the research. SK, PG, SS, AW, ART, KN, VV, SC and NR wrote the manuscript. 


\section{ACKNOWLEDGMENTS}

This work benefited from the assistance of four dedicated interns: N Pricoupenko, S Bekkali, J Lebert and S Diallo. 


\section{REFERENCES}

1. Fawell, S., J. Seery, Y. Daikh, C. Moore, L.L. Chen, B. Pepinsky, and J. Barsoum. 1994. Tat-mediated delivery of heterologous proteins into cells. Proc. Natl. Acad. Sci. U. S. A. 91:664-668.

2. Morris, M.C., L. Chaloin, F. Heitz, and G. Divita. 2000. Translocating peptides and proteins and their use for gene delivery. Curr. Opin. Biotechnol. 11:461-466.

3. Polyakov, V., V. Sharma, J.L. Dahlheimer, C.M. Pica, G.D. Luker, and D. PiwnicaWorms. 2000. Novel Tat-peptide chelates for direct transduction of technetium-99m and rhenium into human cells for imaging and radiotherapy. Bioconjug. Chem. 11:762-771.

4. Torchilin, V.P., R. Rammohan, V. Weissig, and T.S. Levchenko. 2001. TAT peptide on the surface of liposomes affords their efficient intracellular delivery even at low temperature and in the presence of metabolic inhibitors. Proc. Natl. Acad. Sci. U. S. A. 98:8786-8791.

5. Jiao, C.-Y., D. Delaroche, F. Burlina, I.D. Alves, G. Chassaing, and S. Sagan. 2009. Translocation and endocytosis for cell-penetrating peptide internalization. J. Biol. Chem. 284:33957-33965.

6. Bechara, C., and S. Sagan. 2013. Cell-penetrating peptides: 20 years later, where do we stand? FEBS Lett. 587:1693-1702.

7. Binder, H., and G. Lindblom. 2003. Charge-dependent translocation of the Trojan peptide penetratin across lipid membranes. Biophys. J. 85:982-995.

8. Persson, D., P.E.G. Thorén, M. Herner, P. Lincoln, and B. Nordén. 2003. Application of a novel analysis to measure the binding of the membrane-translocating peptide penetratin to negatively charged liposomes. Biochemistry. 42:421-429.

9. Thorén, P.E.G., D. Persson, E.K. Esbjörner, M. Goksör, P. Lincoln, and B. Nordén. 2004. Membrane binding and translocation of cell-penetrating peptides. Biochemistry. 43:34713489.

10. Amand, H.L., C.L. Boström, P. Lincoln, B. Nordén, and E.K. Esbjörner. 2011. Binding of cell-penetrating penetratin peptides to plasma membrane vesicles correlates directly with cellular uptake. Biochim. Biophys. Acta. 1808:1860-1867.

11. Walrant, A., L. Matheron, S. Cribier, S. Chaignepain, M.-L. Jobin, S. Sagan, and I.D. Alves. 2013. Direct translocation of cell-penetrating peptides in liposomes: a combined mass spectrometry quantification and fluorescence detection study. Anal. Biochem. 438:1-10.

12. Wheaten, S.A., F.D.O. Ablan, B.L. Spaller, J.M. Trieu, and P.F. Almeida. 2013. Translocation of cationic amphipathic peptides across the membranes of pure phospholipid giant vesicles. J. Am. Chem. Soc. 135:16517-16525.

13. Swiecicki, J.-M., A. Bartsch, J. Tailhades, M. Di Pisa, B. Heller, G. Chassaing, C. Mansuy, F. Burlina, and S. Lavielle. 2014. The efficacies of cell-penetrating peptides in 
accumulating in large unilamellar vesicles depend on their ability to form inverted micelles. Chembiochem Eur. J. Chem. Biol. 15:884-891.

14. Swiecicki, J.-M., M. Di Pisa, F. Burlina, P. Lécorché, C. Mansuy, G. Chassaing, and S. Lavielle. 2015. Accumulation of cell-penetrating peptides in large unilamellar vesicles: A straightforward screening assay for investigating the internalization mechanism. Biopolymers. 104:533-543.

15. Islam, M.Z., S. Sharmin, V. Levadnyy, S.U. Alam Shibly, and M. Yamazaki. 2017. Effects of Mechanical Properties of Lipid Bilayers on the Entry of Cell-Penetrating Peptides into Single Vesicles. Langmuir ACS J. Surf. Colloids. 33:2433-2443.

16. Herce, H.D., A.E. Garcia, J. Litt, R.S. Kane, P. Martin, N. Enrique, A. Rebolledo, and V. Milesi. 2009. Arginine-rich peptides destabilize the plasma membrane, consistent with a pore formation translocation mechanism of cell-penetrating peptides. Biophys. J. 97:1917-1925.

17. Bayley, H., B. Cronin, A. Heron, M.A. Holden, W.L. Hwang, R. Syeda, J. Thompson, and M. Wallace. 2008. Droplet interface bilayers. Mol. Biosyst. 4:1191-1208.

18. Funakoshi, K., H. Suzuki, and S. Takeuchi. 2006. Lipid bilayer formation by contacting monolayers in a microfluidic device for membrane protein analysis. Anal. Chem. 78:81698174.

19. Huang, J., M. Lein, C. Gunderson, and M.A. Holden. 2011. Direct quantitation of peptidemediated protein transport across a droplet-interface bilayer. J. Am. Chem. Soc. 133:15818-15821.

20. Thiam, A.R., N. Bremond, and J. Bibette. 2012. From stability to permeability of adhesive emulsion bilayers. Langmuir ACS J. Surf. Colloids. 28:6291-6298.

21. Li, X., J. Huang, M.A. Holden, and M. Chen. 2017. Peptide-Mediated Membrane Transport of Macromolecular Cargo Driven by Membrane Asymmetry. Anal. Chem. 89:12369-12374.

22. van Meer, G., D.R. Voelker, and G.W. Feigenson. 2008. Membrane lipids: where they are and how they behave. Nat. Rev. Mol. Cell Biol. 9:112-124.

23. Derossi, D., A.H. Joliot, G. Chassaing, and A. Prochiantz. 1994. The third helix of the Antennapedia homeodomain translocates through biological membranes. J. Biol. Chem. 269:10444-10450.

24. Magzoub, M., L.E.G. Eriksson, and A. Gräslund. 2002. Conformational states of the cellpenetrating peptide penetratin when interacting with phospholipid vesicles: effects of surface charge and peptide concentration. Biochim. Biophys. Acta. 1563:53-63.

25. Balayssac, S., F. Burlina, O. Convert, G. Bolbach, G. Chassaing, and O. Lequin. 2006. Comparison of penetratin and other homeodomain-derived cell-penetrating peptides: interaction in a membrane-mimicking environment and cellular uptake efficiency. Biochemistry. 45:1408-1420. 
26. Caesar, C.E.B., E.K. Esbjörner, P. Lincoln, and B. Nordén. 2006. Membrane interactions of cell-penetrating peptides probed by tryptophan fluorescence and dichroism techniques: correlations of structure to cellular uptake. Biochemistry. 45:7682-7692.

27. Walrant, A., I. Correia, C.-Y. Jiao, O. Lequin, E.H. Bent, N. Goasdoué, C. Lacombe, G. Chassaing, S. Sagan, and I.D. Alves. 2011. Different membrane behaviour and cellular uptake of three basic arginine-rich peptides. Biochim. Biophys. Acta. 1808:382-393.

28. Gross, L.C.M., A.J. Heron, S.C. Baca, and M.I. Wallace. 2011. Determining membrane capacitance by dynamic control of droplet interface bilayer area. Langmuir ACS J. Surf. Colloids. 27:14335-14342.

29. Walde, P., K. Cosentino, H. Engel, and P. Stano. 2010. Giant vesicles: preparations and applications. Chembiochem Eur. J. Chem. Biol. 11:848-865.

30. Ben M'barek, K., D. Ajjaji, A. Chorlay, S. Vanni, L. Forêt, and A.R. Thiam. 2017. ER Membrane Phospholipids and Surface Tension Control Cellular Lipid Droplet Formation. Dev. Cell. 41:591-604.e7.

31. Hauser, H., and G.G. Shipley. 1984. Interactions of divalent cations with phosphatidylserine bilayer membranes. Biochemistry. 23:34-41.

32. Mao, Y., Y. Du, X. Cang, J. Wang, Z. Chen, H. Yang, and H. Jiang. 2013. Binding competition to the POPG lipid bilayer of $\mathrm{Ca} 2+, \mathrm{Mg} 2+, \mathrm{Na}+$, and $\mathrm{K}+$ in different ion mixtures and biological implication. J. Phys. Chem. B. 117:850-858.

33. Devaux, P.F., and R. Morris. 2004. Transmembrane asymmetry and lateral domains in biological membranes. Traffic Cph. Den. 5:241-246.

34. Hwang, W.L., M. Chen, B. Cronin, M.A. Holden, and H. Bayley. 2008. Asymmetric droplet interface bilayers. J. Am. Chem. Soc. 130:5878-5879.

35. Di Pisa, M., G. Chassaing, and J.-M. Swiecicki. 2015. Translocation mechanism(s) of cellpenetrating peptides: biophysical studies using artificial membrane bilayers. Biochemistry. 54:194-207.

36. Kauffman, W.B., T. Fuselier, J. He, and W.C. Wimley. 2015. Mechanism Matters: A Taxonomy of Cell Penetrating Peptides. Trends Biochem. Sci. 40:749-764. 

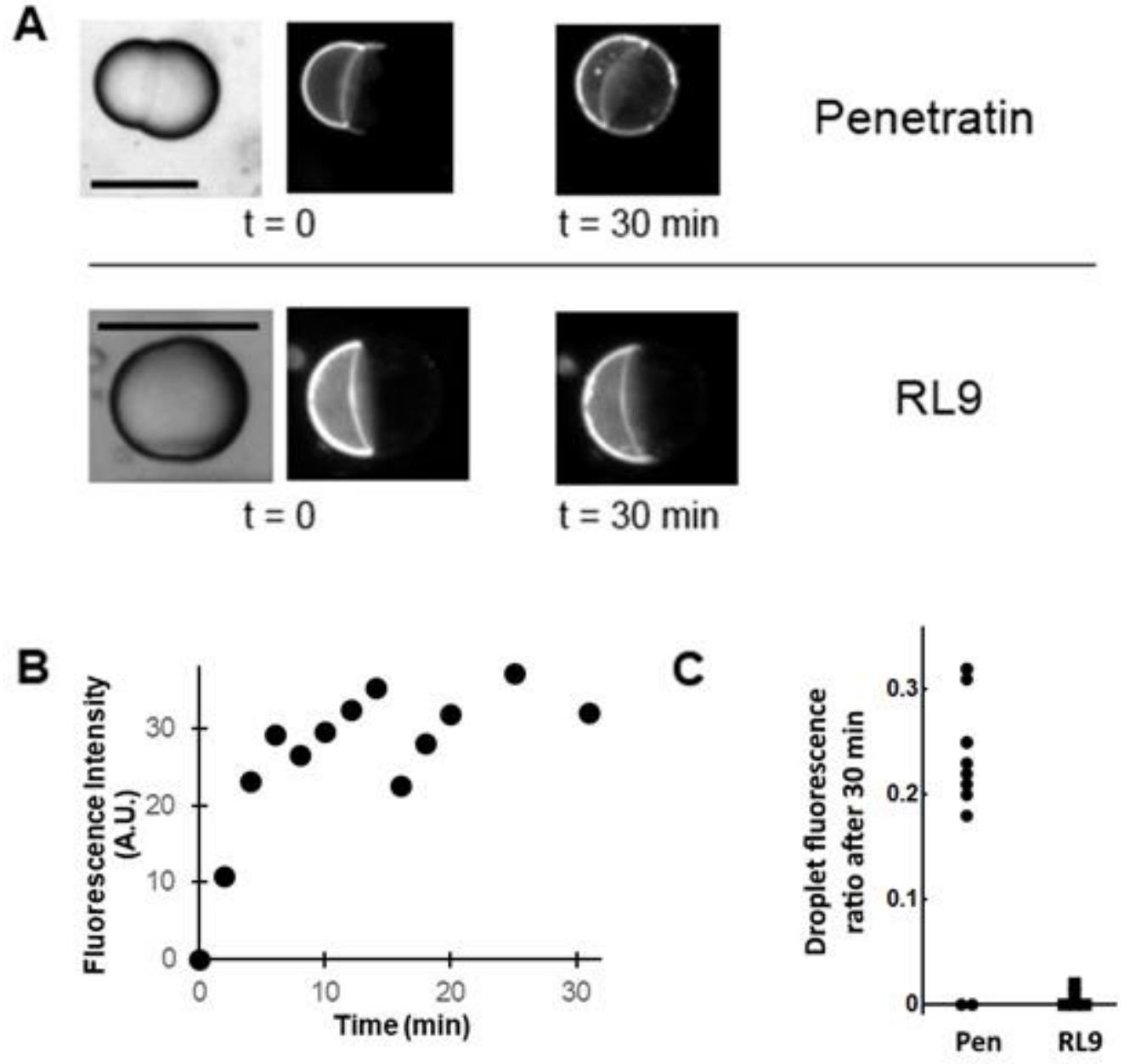

A: Bright field and fluorescence microscopy images of a pair of droplets with a POPG bilayer at their interface. At $\mathrm{t}=0$ fluo-penetratin is located in the left droplet only. After 30 minutes some of the fluo-penetratin has crossed the DIB. An increase of the contact area between the droplets is detected during the timecourse of the experiment. In the case of fluo-RL9 $(5 \mu \mathrm{M})$, there is no measurable passage over a 30 minutes time scale. Scale bar lengths are $50 \mu \mathrm{m}$.

B: Time course of the measured fluorescence intensity in the initially fluo-penetratin free droplet. The apparent drop of concentration at $\mathrm{t}=16 \mathrm{~min}$ may be artifactual due to the occurrence of a bright spot inside the distal droplet. The raising of the fluorescence during the first 10 minutes of the experiment is the signature of the translocation of some fluo-penetratin through the POPG DIB towards the initially peptide free droplet.

C: Ratio of fluorescence intensities between the initially peptide free droplet and the peptide source droplet after 30 minutes. Each symbol corresponds to one experiment. "Pen" stands for fluo-penetratin and "RL9" for fluo-RL9. 
Figure 2: Impact of the lipid composition on the translocation probability of fluopenetratin

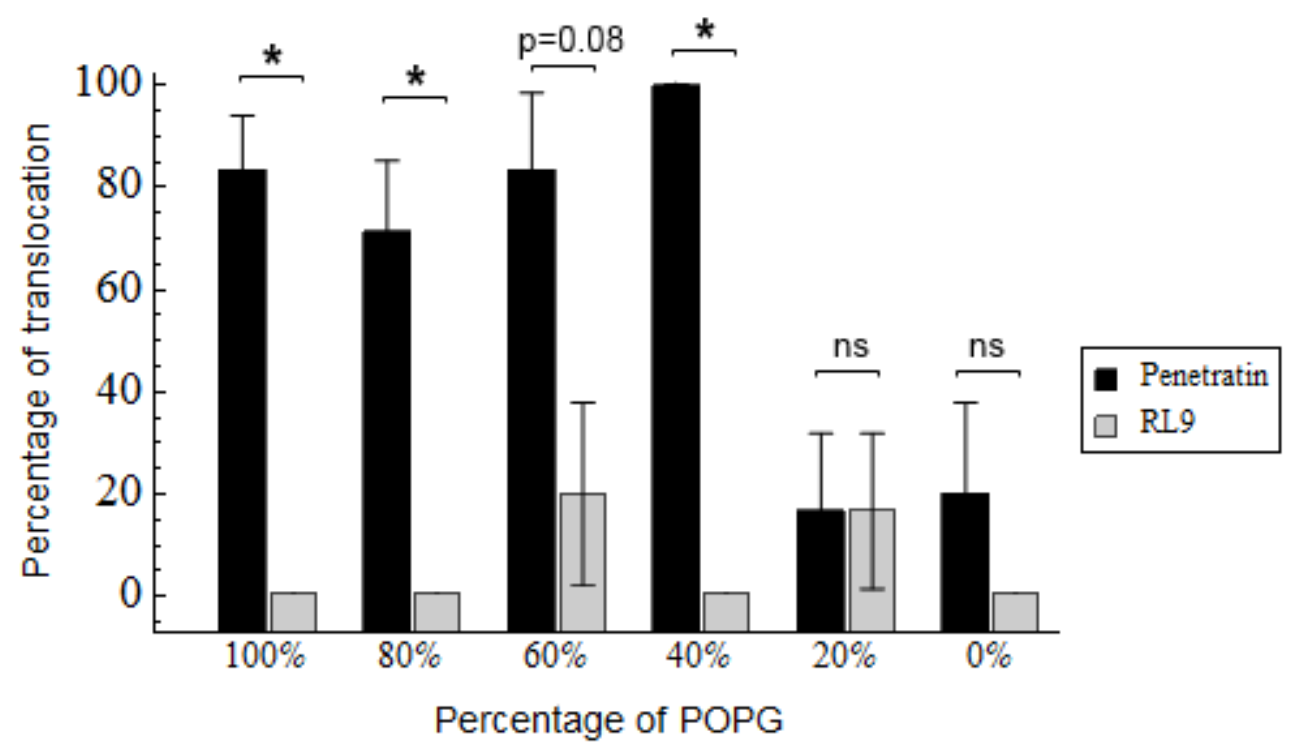

The translocation is tested for different percentage of POPG mixed with POPC. For each composition the probability of the occurrence of translocation is compared between the penetratin and RL9 peptide. The percentage of translocation is the percentage of experiments for which a significant translocation is observed. The significance criterion is $\mathrm{p}<0.05$ with an exact fisher test. 5 to 12 experiments have been conducted for each condition (a condition corresponding to a type of peptide and a lipid composition). 
Figure 3: Crossing of a soluble fluorescent probe FP488 through DIBs

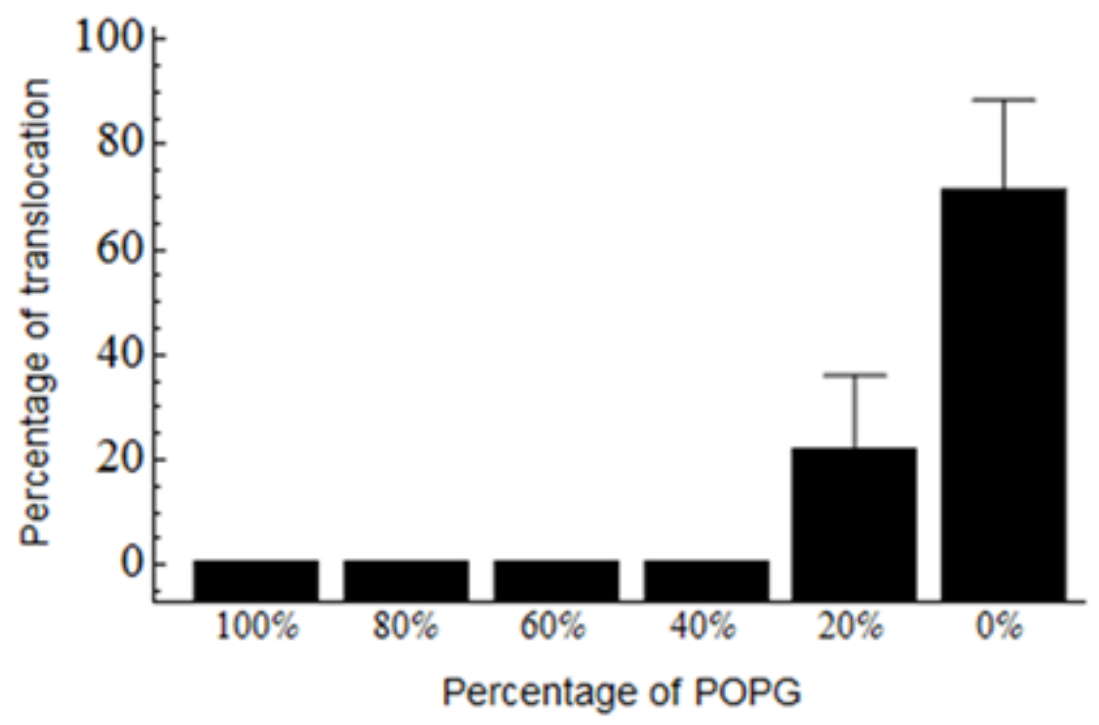

Crossing of bare FP 488 through POPC/POPG DIBs with different ratios. Crossing was consistently observed for $100 \%$ POPC DIBs. No such crossing was detected for DIBs with more than $40 \%$ POPG. 
Figure 4: Impact of the concentration of fluo-penetratin on its translocation probability

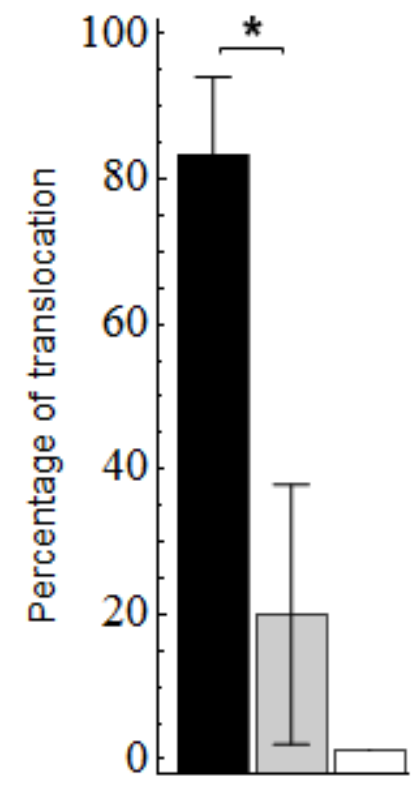

- Penetratin $5 \mu \mathrm{M}$

$\square$ Penetratin $2 \mu \mathrm{M}$

$\square$ RL9 $5 \mu \mathrm{M}$

The percentage of experiments for which translocation is observed is significantly reduced when fluo-penetratin concentration is lowered from $5 \mu \mathrm{M}$ to $2 \mu \mathrm{M}$. $\mathrm{n}=5$ to 12 for each condition. 
Figure 5: No translocation of fluo-penetratin through POPS DIB is observed

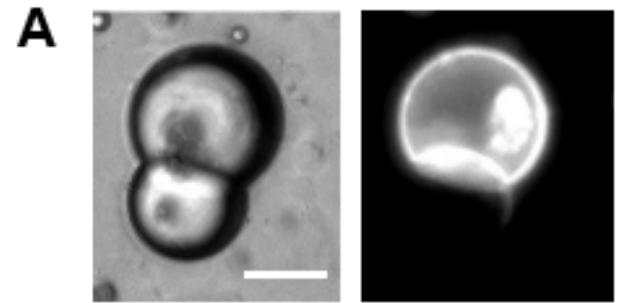

$t=0$

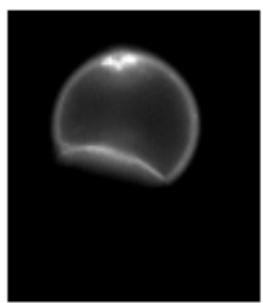

$\mathrm{t}=30 \mathrm{~min}$

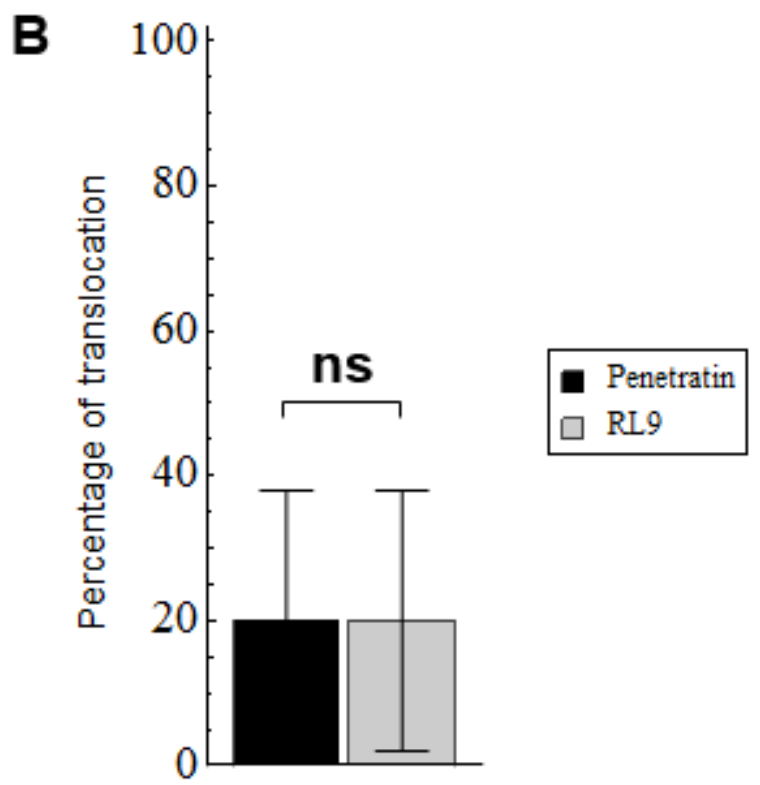

A: The DIB and monolayers surrounding the droplets are 100\% POPS. Fluo-penetratin did not translocate. Scale bar length is $20 \mu \mathrm{m}$.

B: In most experiments fluo-penetratin did not translocate. It showed no better cell-penetration ability than fluo-RL9. 
Figure 6: The asymmetry of the DIB is maintained over $\sim 45$ min
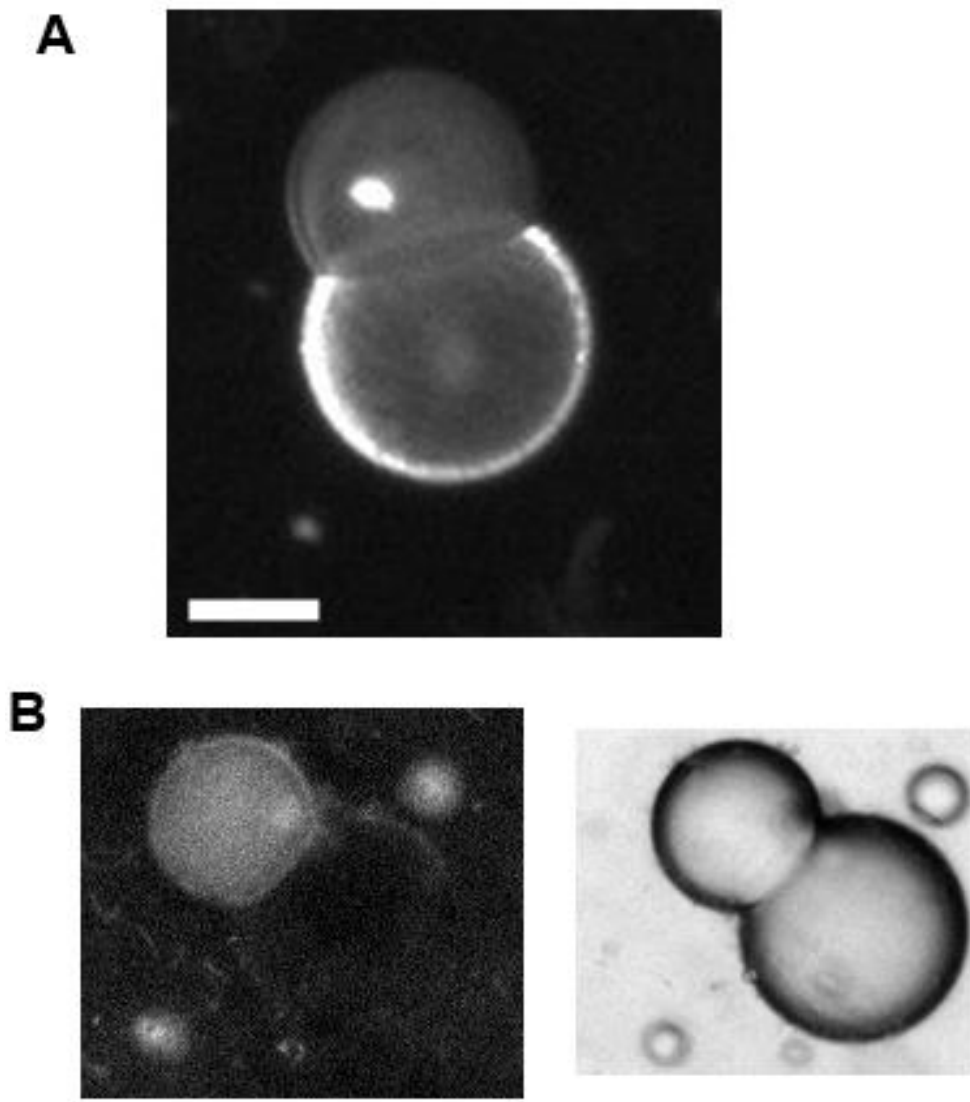

\section{$\mathrm{t}=0 \mathrm{~min}$}
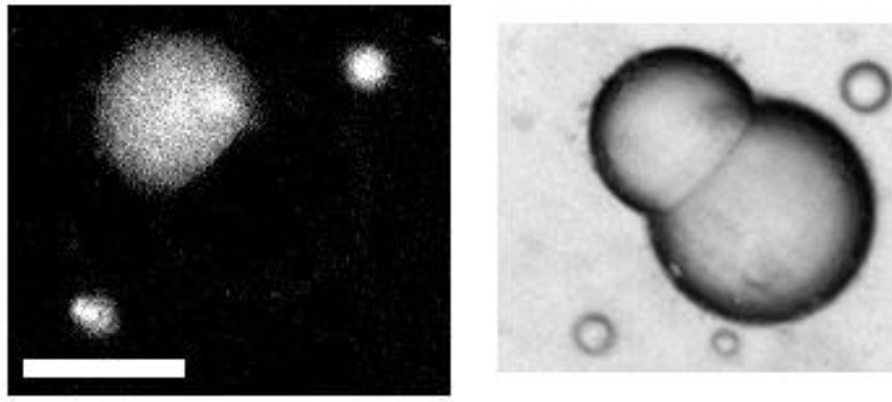

$t=45 \min$

A: A pair of droplets covered with DOPC for the upper one and DOPS for the lower one. Both contain fluo-annexin V. The monolayer of the DOPC droplet is not visible whereas the monolayer of the DOPS droplet is clearly visible.

B: A pair of droplet was observed for $45 \mathrm{~min}$. The upper droplet was initially surrounded by DOPC lipids and filled with fluo-annexin V. The lower droplet was initially surrounded by DOPS lipids and free from fluo-annexin V. A slight fluorescence visible on a part of the edge of the lower droplet is likely due to the presence of a bright droplet in its vicinity (because the lower droplet is free from fluo-annexin V). No transfer of DOPS from the lower to the upper droplet was detected within a 45 minutes time scale. To make sure to be able to detect a possible fluorescence of the edges of the upper droplet after 45 minutes, the contrast of the picture has been enhanced. Scale bar length is $50 \mu \mathrm{m}$. 
Figure 7: No translocation of fluo-penetratin was observed through asymmetric POPG:POPC bilayers, whatever the initial side location of fluo-penetratin

A

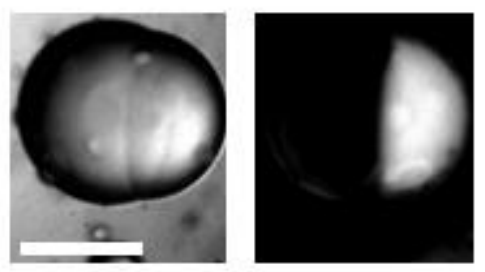

$t=0$

B

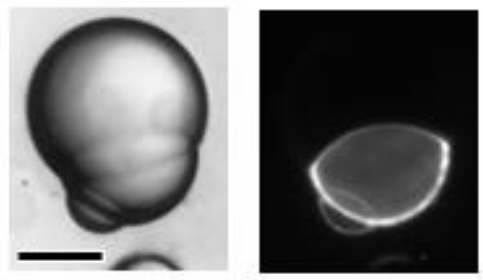

$t=0$

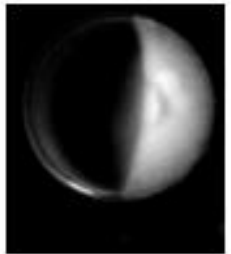

$\mathrm{t}=30 \mathrm{~min}$

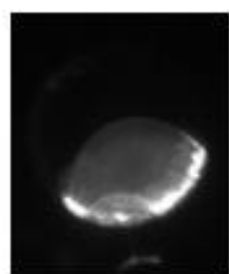

$\mathrm{t}=30 \mathrm{~min}$

A: The left droplet was covered with POPG, the right one with POPC. The DIB was thus asymmetric. Fluo-penetratin $(5 \mu \mathrm{M})$ was initially present in the POPC side of the DIB and did not translocate. Scale bar represents $300 \mu \mathrm{m}$.

B: The lower droplet was covered with POPG, the upper one with POPC. The DIB was thus asymmetric. Penetratin $(5 \mu \mathrm{M})$ was initially present in the POPG side of the DIB and did not translocate. Scale bar length is $50 \mu \mathrm{m}$. 
Figure 8: Fluo-penetratin translocates through proximal:distal POPG:POPS DIBs but not through proximal:distal POPS:POPG DIBs

A
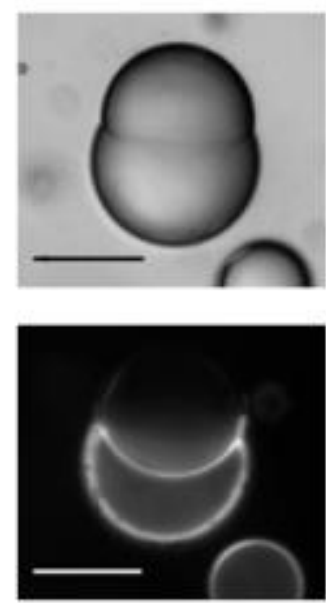

$\mathrm{t}=0 \mathrm{~min}$

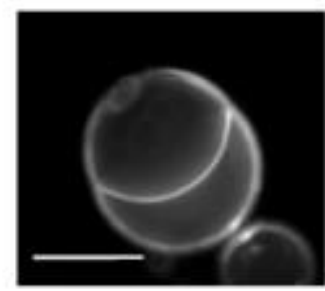

$\mathrm{t}=30 \mathrm{~min}$
B
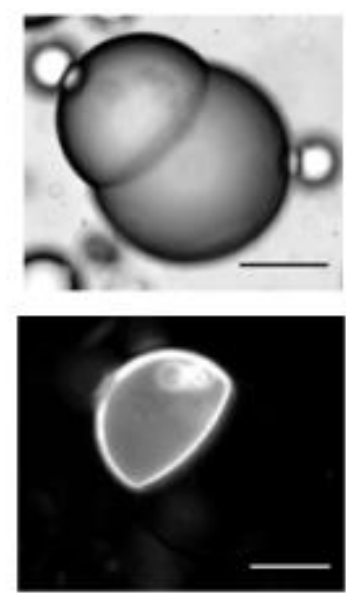

$\mathrm{t}=0 \mathrm{~min}$

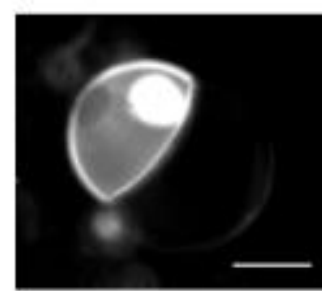

$\mathrm{t}=30 \mathrm{~min}$

C

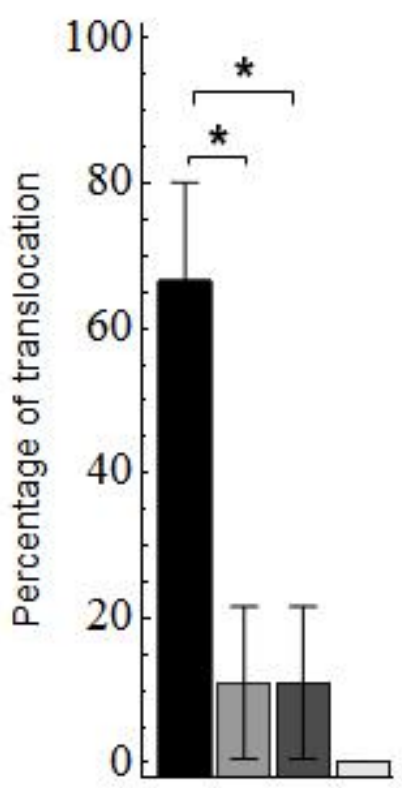

Penetratin prox:dist POPG:POPS

RL9 prox:dist POPG:POPS

- Penetratin prox:dist POPS:POPG

RL9 prox:dist POPS:POPG

A: Translocation of fluo-penetratin $(5 \mu \mathrm{M})$ through a proximal:distal POPG:POPS DIB. Scale bar corresponds to $40 \mu \mathrm{m}$.

B: Absence of translocation of fluo-penetratin $(5 \mu \mathrm{M})$ through a proximal:distal POPS:POPG DIB. Scale bar corresponds to $40 \mu \mathrm{m}$.

C: Fluo-penetratin $(5 \mu \mathrm{M})$ translocates significantly through proximal:distal POPG:POPS DIBs $(n=12)$ as compared to fluo-RL9 $(n=5)$. This translocation of fluo-penetratin is significantly more frequent than the translocation of fluo-penetratin through proximal:distal POPS:POPG DIBs $(n=9)$. 
Table 1: No translocation of fluo-penetratin is observed through asymmetric POPG:POPC or POPC:POPG bilayer

\begin{tabular}{|l|l|l|l|l|}
\hline $\begin{array}{l}\text { DIB leaflet } \\
\text { composition : }\end{array}$ & $\begin{array}{l}\text { Number of } \\
\text { experiments with } \\
\text { translocation of } \\
\text { fluo-penetratin }\end{array}$ & $\begin{array}{l}\text { Number of } \\
\text { experiments } \\
\text { with no } \\
\text { translocation } \\
\text { of fluo- } \\
\text { penetratin }\end{array}$ & $\begin{array}{l}\text { Number of } \\
\text { experiments } \\
\text { with } \\
\text { translocation } \\
\text { of fluo-RL9 }\end{array}$ & $\begin{array}{l}\text { Number of } \\
\text { experiments } \\
\text { with no } \\
\text { translocation } \\
\text { of fluo-RL9 }\end{array}$ \\
\hline $\begin{array}{l}\text { POPC on the } \\
\text { proximal leaflet, } \\
\text { POPG on the other } \\
\text { leaflet (distal) }\end{array}$ & 0 & 6 & 0 & 5 \\
\hline $\begin{array}{l}\text { POPG on the } \\
\text { proximal leaflet, } \\
\text { POPC on the other } \\
\text { leaflet (distal) }\end{array}$ & 1 & 5 & 1 & 5 \\
\hline
\end{tabular}


Supplementary figure S1: kinetics of fluo-penetratin translocation for different lipid DIB composition

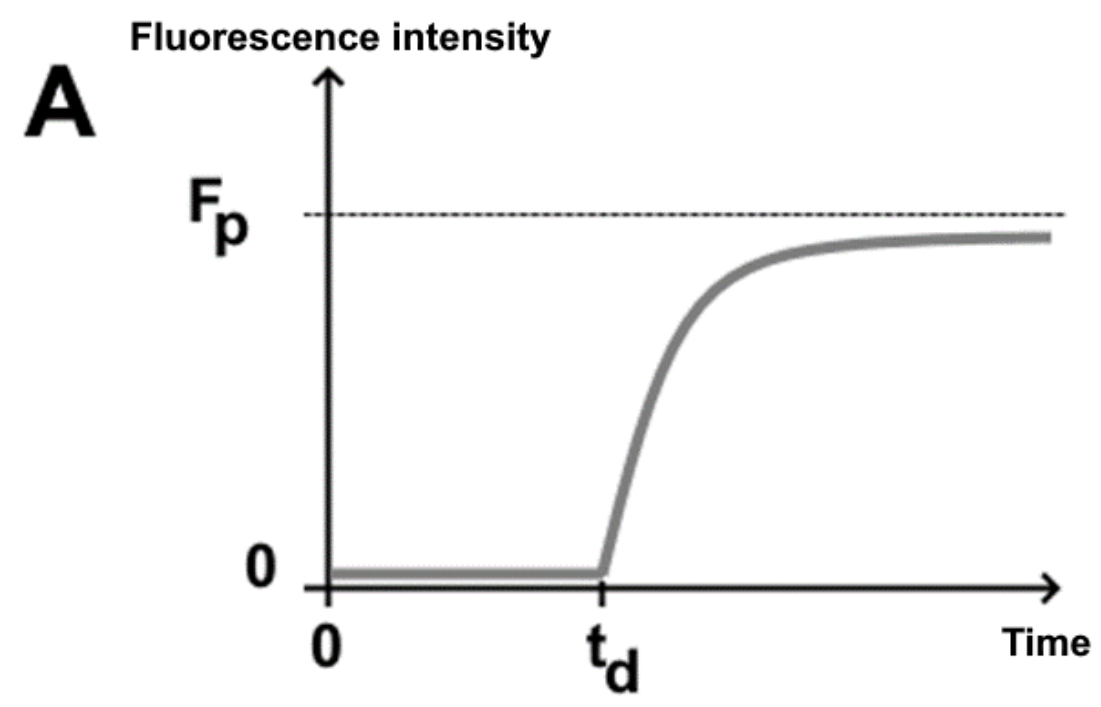

B

\begin{tabular}{|c|c|c|c|c|c|c|c|c|c|}
\hline \multirow{2}{*}{ DIB composition } & \multicolumn{3}{|c|}{$\mathrm{t}_{\mathrm{d}}$ (minutes) } & \multicolumn{2}{c|}{$\tau$ (minutes) } & \multicolumn{3}{c|}{$F_{p} / F_{S}$} \\
\cline { 2 - 10 } & mean & $\min$ & $\max$ & mean & $\min$ & $\max$ & mean & $\min$ & $\max$ \\
\hline $\begin{array}{c}\text { Symmetric } \\
\text { 100\%POPG }\end{array}$ & 3 & 0 & 19 & 7 & 1 & 17 & 0.23 & 0.20 & 0.25 \\
\hline $\begin{array}{c}\text { Symmetric } \\
\text { POPG/POPC 4/1 }\end{array}$ & 9 & 0 & 25 & 5 & 5 & 6 & 0.32 & 0.30 & 0.35 \\
\hline $\begin{array}{c}\text { Symmetric } \\
\text { POPG/POPC 3/2 }\end{array}$ & 0 & 0 & 0 & 1 & 1 & 2 & 0.31 & 0.25 & 0.38 \\
\hline $\begin{array}{c}\text { Symmetric } \\
\text { POPG/POPC 2/3 }\end{array}$ & 5 & 0 & 20 & 8 & 2 & 14 & 0.11 & 0.10 & 0.12 \\
\hline $\begin{array}{c}\text { Asymmetric } \\
\text { POPG(proximal): } \\
\text { POPS(distal) }\end{array}$ & 1 & 0 & 5 & 6 & 3 & 10 & 0.30 & 0.12 & 0.54 \\
\hline
\end{tabular}

We have performed an analysis of the kinetics of the fluo-penetratin translocation for the 5 DIB compositions for which translocation was the predominant observed behavior. Half of these experiments could be well fitted with the following function for the fluorescence intensity of the initially peptide-free droplet (figure A):

$$
\begin{aligned}
& F(t)=0 \text { if } t<t_{d} \\
& F(t)=F_{p}\left(1-\exp \left(-\left(t-t_{d}\right) / \tau\right)\right) \text { if } t \geq t_{d}
\end{aligned}
$$


$t_{d}$ is the delay for the onset of translocation.

$\tau$ is the crossing characteristic time.

$F_{p}$ is the fluorescence intensity at the plateau.

Half of the experiments could not be fitted with this function for one of these reasons: (i) Not enough data points had been gathered through time (ii) The plateau was not clearly reached at the end of the acquisition (iii) Translocation had slightly started before the first observation point which prevented to measure the full kinetics. The parameters obtained by fitting of the fluorescence intensity kinetics are summarized on figure B. For each parameter, the mean value obtained over all the experiments for this DIB composition is given. As an illustration of the variability of the fitted parameters the extreme values (minimum and maximum) obtained for each condition are also given.

It is an interesting feature of these kinetics that a delay (quantified by $t_{d}$ ) is sometimes observed before the onset of the translocation. This may indicate a time necessary for the formation of a transient peptide-lipide structure that will drive the translocation. It is consistent with a stochastic formation of this structure. The characteristic time $\tau$ of the translocation process is always of the order of a few minutes for all DIB compositions but a great dispersion of its precise value is observed (ranging from 1 minute to 17 minutes). This may indicate a certain variability of the peptide-lipid structures that will lead to the translocation. The fluorescence plateau $\mathrm{F}_{\mathrm{p}}$ is quantified relatively to the fluorescence intensity of the source droplet initially containing the fluo-penetratin $\mathrm{F}_{\mathrm{S}}$. It is noteworthy that despite a certain variability this plateau is generally observed at a $\sim 0.2$ ratio which indicates that the equilibrium between the source and the target droplet is not reached. Thus it appears that in our experimental conditions we also evidenced a transient behavior of the structure enabling translocation. 
Supplementary figure S2: sensitivity of DOPS detection with fluo-annexin V

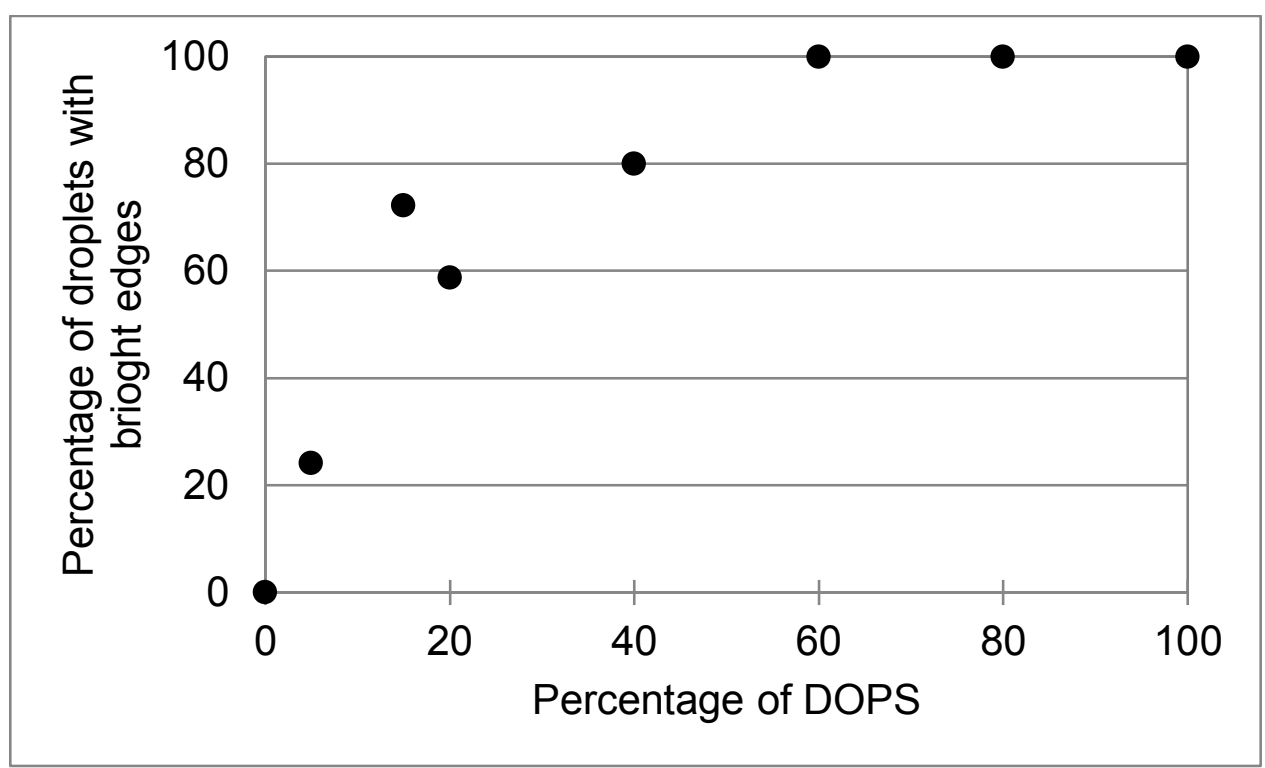

Different populations of droplets with various percentages of DOPS (in DOPC) containing fluoannexin $\mathrm{V}$ were made. The percentage of droplets the monolayers of which were clearly brighter than the bulk was quantified. From this study it appears that fluo-annexin V is sensitive enough to detect $\sim 10 \%$ of DOPS in a monolayer. 


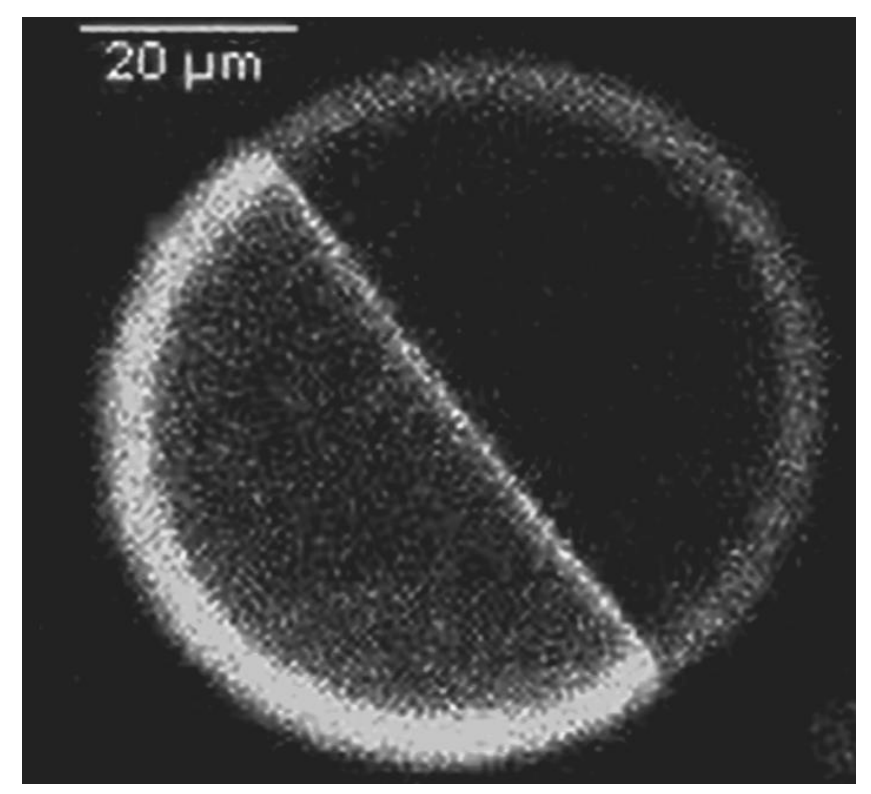

A confocal microscopy image of a pair of droplets with a symmetric POPG DIB is shown. The left droplet was initially free of fluo-penetratin. The image is taken at $t=10 \mathrm{~min}$. It shows that both in the initially peptide free droplet and in the reservoir droplet a significant enrichment of fluo-penetratin is observed on the edges (monolayers and DIB) and that some peptides also remain in the aqueous bulk of the droplets. 